\title{
Performance measurement of a new concept reciprocating piston expander (RPE) using a newly developed small-scale dynamometer unit
}

\author{
MNA Mohamad ${ }^{1},{ }^{*}$, MF Hushim², WNAW Muhammad ${ }^{2}$, A Razali $^{1}, \mathrm{~A} \mathrm{Khalid}^{2}$, and MF \\ Zakaria $^{3}$ \\ ${ }^{1}$ Faculty of Mechanical and Manufacturing Engineering, Universiti Tun Hussein Onn \\ Malaysia, 86400 Parit Raja, Batu Pahat, Johor, Malaysia. \\ ${ }^{2}$ Faculty of Engineering Technology, Universiti Tun Hussein Onn Malaysia, 86400 Parit \\ Raja, Batu Pahat, Johor, Malaysia. \\ ${ }^{3}$ Faculty of Electronic and Electric Engineering, Universiti Tun Hussein Onn Malaysia, \\ 86400 Parit Raja, Batu Pahat, Johor, Malaysia. \\ E-mail: anuarm@uthm.edu.my
}

\begin{abstract}
This paper presents the progress of a small-scale dynamometer prototype development for performance measurement of a reciprocating piston expander (RPE). Since the available dynamometer systems in the market are limited to specific applications that require for the customization, their price normally very expensive. Since the current study on the RPE required a dynamometer unit, therefore, a new and cheaper dynamometer prototype that was suitable for RPE application has been developed. Using air as RPE working fluid, a case study has been carried out to measure its performance at different inlet fluid conditions, i.e., within $20^{\circ} \mathrm{C}-140^{\circ} \mathrm{C}$ and 3-5 bars. The results observed that the performance of RPE was proportionally increased to the increased of inlet fluid pressure and temperature. The maximum brake power produced was 27 Watt when the RPE operated at $140^{\circ} \mathrm{C}, 5$ bars and the speed of $820 \mathrm{rpm}$. It also revealed that the changes in the pressure of inlet fluid can give significant change on the performance of the RPE due to its direct relation to the RPE actual rotating force. Although the RPE and dynamometer seem good being adapted to each other, both of them require some improvements to ensure both systems well operated and reliable.
\end{abstract}

\section{Introduction}

Most of the environmental problems faced by the world today caused by vast consumption of fossil fuel in order to meet the current energy demands [1]. Although there are efficient energy conversion systems have investigated and introduced, the

\footnotetext{
*Corresponding author: anuarm@uthm.edu.my
} 
fuel consumptions can no longer be reduced when the design points have been met [2], there are still the methods to increase the overall efficiency of the system with fewer fuels and lessen the pollutions. Besides, the introduction of cleaner fuels and replacement or modification of the energy conversion systems being a solution to meet the above objectives, recovering the leftover energy especially in terms of wasted heat into a useful energy has been considered and proposed as the best solution [3],[4]. Among the heat recovery systems, recovering through an Organic Rankine Cycle (ORC) system is a viable method since the ability of this system to recover the most abundant amount of heat at low to medium temperature heat [5] and for small-scale applications [6].

Although the ORC system seems feasible, it has a limitation on its critical component, i.e., its expander as it is normally designed for certain operating conditions. In order to evaluate the performance of the ORC systems, especially during the development of its expander unit, there is a system that is used to measure the power output delivered by the expander at a predetermined condition. Most of the power driving unit such as engines, motors, transmissions, generators, turbines, and expanders require a dynamometer system to measure the power, torque, and speed from the rotating force of the driving unit by introducing variable loading conditions on the driving unit tested across the range of engine speeds and durations [7]. When a driving unit operates, it generates the potential and kinetic energy thus rotates its drive shaft. As a load absorbing unit, the dynamometer absorbs the energy through a braking action, while at the same time it measures the performance of the driving unit. Although the dynamometer system is commercially available equipment, its price is very expensive since the system has been customized to suit the requirement for the overall test system. Some of the ready-made dynamometers such as vehicle or engine dynamometers are designed for a specific purpose, i.e., to measure the performance of the engines that operate at the specific operational range, and of course, these dynamometers are bulky and not suitable for small scale driving unit measurement.

Since the dynamometer is a crucial system while developing the expander for the performance measurement, with the limited resources and funds, it is wise to develop own dynamometer system that suitable and can operate at acceptable conditions. Therefore, this paper presents the progress on developing a prototype of a new small-scale type dynamometer for performance measurement of the RPE unit. To ensure the operation of this prototype met the requirements, a case study has been carried out by measuring the actual RPE performance at different operating conditions. The weaknesses and improvements ideas for the dynamometer were also discussed at the end of this paper.

\section{Design and development of a small-scale dynamometer prototype}

Nowadays, there are many types of dynamometers used in the industries, research institutes, service agencies, etc. with some few advantages and disadvantages for each of them based on its counterparts. Although they look different, their designs are originated from the commonly classified dynamometer types such as fluid (water or air) brake, eddy current, prony brake, and cradled generator type dynamometers [8]. Here, the prony brake dynamometer is considered as the simplest design for measuring power output (brake power) and could be potentially developed by anyone at low cost compared to the other type dynamometers. Based on prony brake dynamometer, in this study, the prototype of a smallscale dynamometer that has been developed and its design is shown in figure 1 .

Based on prony brake principle, the rotating force (brake power) of a driving equipment is measured by applying the frictional load in attempt to stop the rotation of the shaft equipment by mean of braking action by the shaft supporting arm that is attached to the load. The supporting arm is normally fixed with the braking mechanism (normally at one 
end) such as brake block or pad that is attached to the shaft. When the load or force gradually applied on the other end side of the arm, the brake mechanism gives the friction to the rotating shaft until as it tries to stop the shaft. During this condition, the brake power is calculated as

$$
P_{b}=2 \pi N T
$$

where, $\quad N$ is angular velocity in revolution per second (rps), and $T$ is the torque applied to the shaft $(\mathrm{Nm})$.

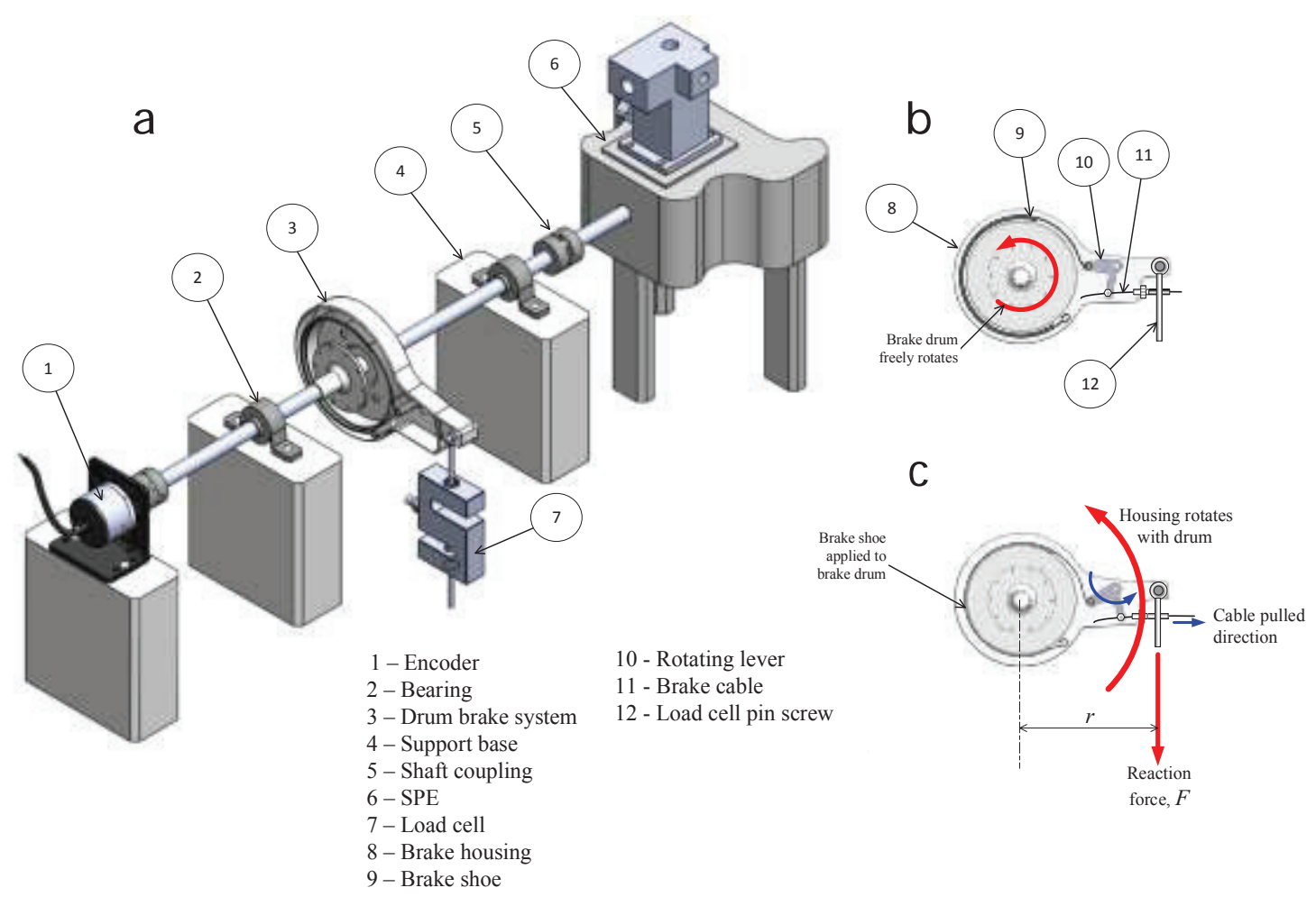

Figure 1. (a) Design layout of a small-scale dynamometer prototype, (b) drum brake condition before load applied and (d) drum brake after load applied.

This type dynamometer was utilized to measure the performance by absorbing the brake power produced by the RPE. From the figure 1, the RPE rotating shaft transferred its power to the dynamometer through the shaft coupling to the drum brake system, i.e., the critical component that enables whether the load has been applied (or not) to the shaft. If the load was not applied, then the brake drum, i.e., the component that has been fixed to the shaft was freely rotates as shown in figure 1(b). The condition if the load applied to the shaft happen when the brake shoe has been forced to rub the drum which caused the entire drum brake system especially its housing to rotate together with the drum. The more force applied to the drum, the more load was given to the RPE shaft, thus the more torque can be measured by multiplying the reaction force, $F$ to the distance, $r$ of the force and the center of the shaft. The torque, $T$ is written as 


$$
T=F r
$$

\section{Case study: Performance measurement of the RPE}

\subsection{The reciprocating piston expander (RPE)}

The expander that has been investigated in this study was a newly developed expander by modifying a few parts of a small two-stroke grass cutter engine. The spark plug port has been replaced with the inlet port of the RPE while the inlet and scavenging ports of the engine have been closed by replacing the original cylinder block of the engine with the new block for the RPE. Therefore, the operational characteristics of the RPE were different from the engine with high pressure working fluid entered the RPE through the spark plug port from the top and the expansion process occurred not by the combustion process but due to highpressure fluid pushed the piston downward. The high-pressure fluid entrance to the RPE was controlled by the valve control switches which was also introduced in [9] to ensure the opening and closing of the solenoid valves happened at the right timing. Figure 2 shows the conversion of the engine into the RPE unit.

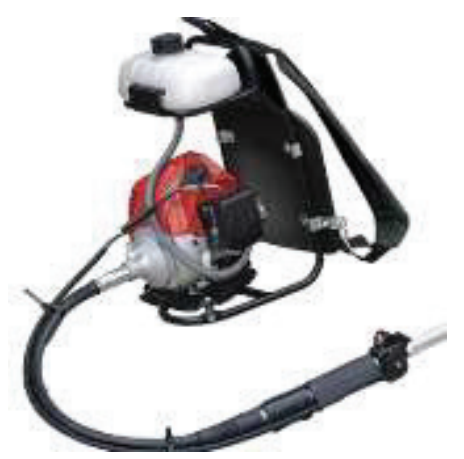

Grass Cutter Engine

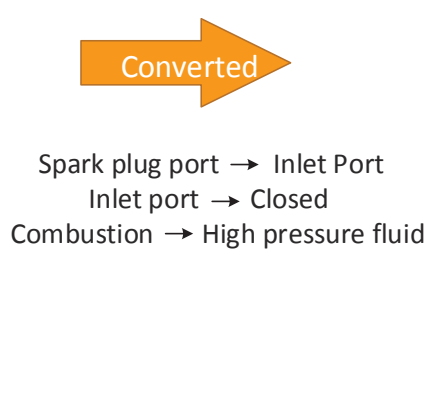

Inlet port $\rightarrow$ Closed Combustion $\rightarrow$ High pressure fluid

Figure 2. Conversion of the small two-stroke engine into RPE.

\subsection{Performance test of the RPE}

The experimental test system for the RPE performance measurement is shown in figure 3. In this study, there were five fluid inlet pressures, (i.e., 3, 3.5, 4, 4.5 and 5 bars) with five temperature conditions (i.e., $20^{\circ} \mathrm{C}, 80^{\circ} \mathrm{C}, 100^{\circ} \mathrm{C}, 120^{\circ} \mathrm{C}$ and $140^{\circ} \mathrm{C}$ ) have been tested for the RPE performance. Here, the compressed air was used as working fluid to operate the expander. The air was supplied from the air compressor unit through a boiler before entering the RPE. 


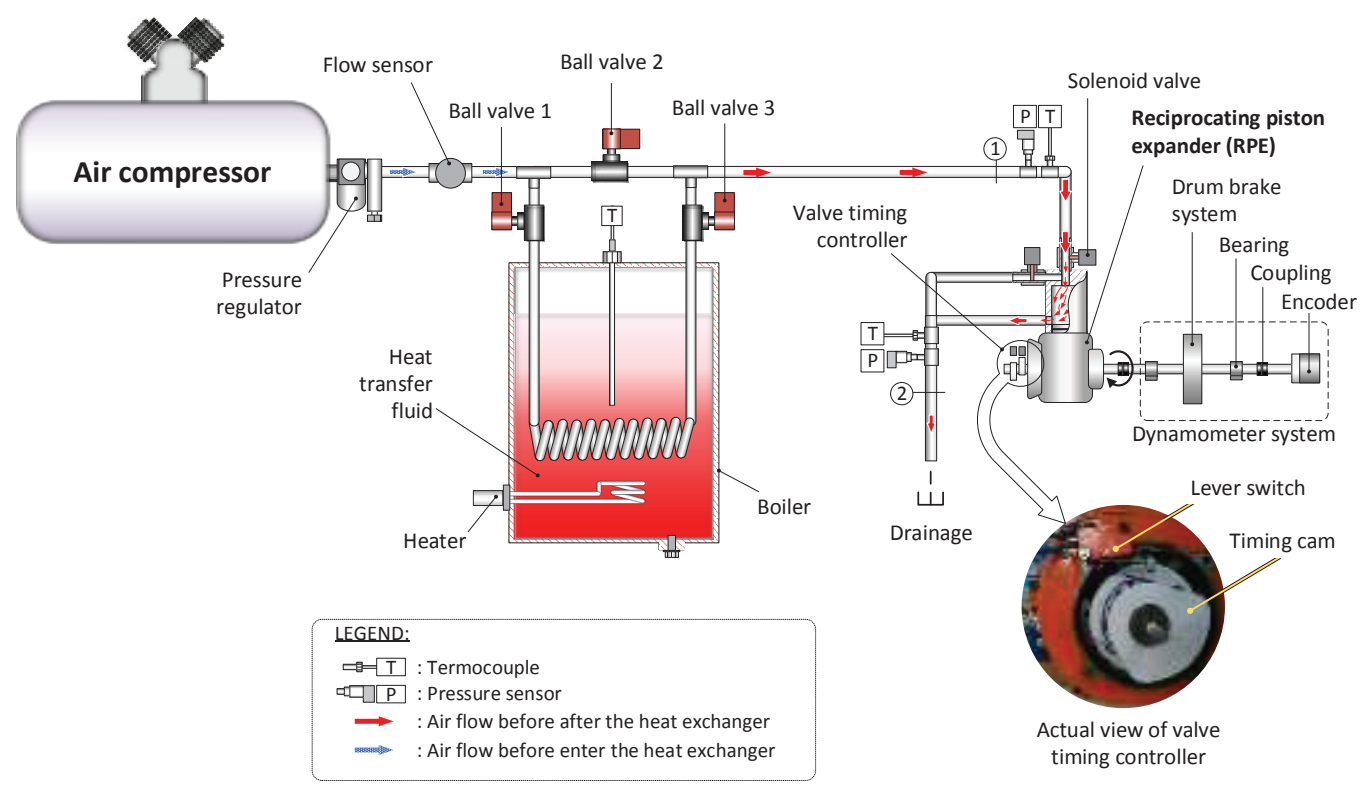

Figure 3. Experimental test system for RPE performance measurement.

In the only case where the RPE operation condition was at room temperature $\left(20^{\circ} \mathrm{C}\right)$, the air bypassed the boiler when the ball valves 1 and 3 closed, but the ball valve 2 was kept open. For the other cases $\left(80^{\circ} \mathrm{C}-140^{\circ} \mathrm{C}\right)$, the air flowed through ball valve 1 , the boiler, the ball valve 3 and the RPE, when the valve 3 was closed but both valves 1 and 3 were opened. The thermodynamics properties of the air at RPE inlet and outlet were measured by thermocouples and pressure sensors, while the performance (i.e., rotational speed, force, torque, and power) were measured through the dynamometer system. This figure also showed the valve control system that was used in this study by adapting the lever switches and timing cams in order to control the right opening and closing angle of the inlet and exhaust valves. In this study, the best timing for inlet and exhaust valves have been determined as $80^{\circ}$ after top dead center (TDC) and $100^{\circ}$ after bottom dead center (BDC), respectively.

\section{Results and discussions}

The study observed that the performance of the RPE was increased with the increase of the pressure and temperature of the inlet fluid. The rotational speed varied at the lowest range within 440-586 rpm when the RPE operated at room temperature $\left(20^{\circ} \mathrm{C}\right)$ and 3 bars of pressure, while at the highest temperature $\left(140^{\circ} \mathrm{C}\right)$ and pressure of 5 bars, the speed varied at the highest range within $820-1075 \mathrm{rpm}$. Figure 4 shows the rotational speed of the RPE when it was tested at no load and full load conditions with different inlet pressure and temperature. 


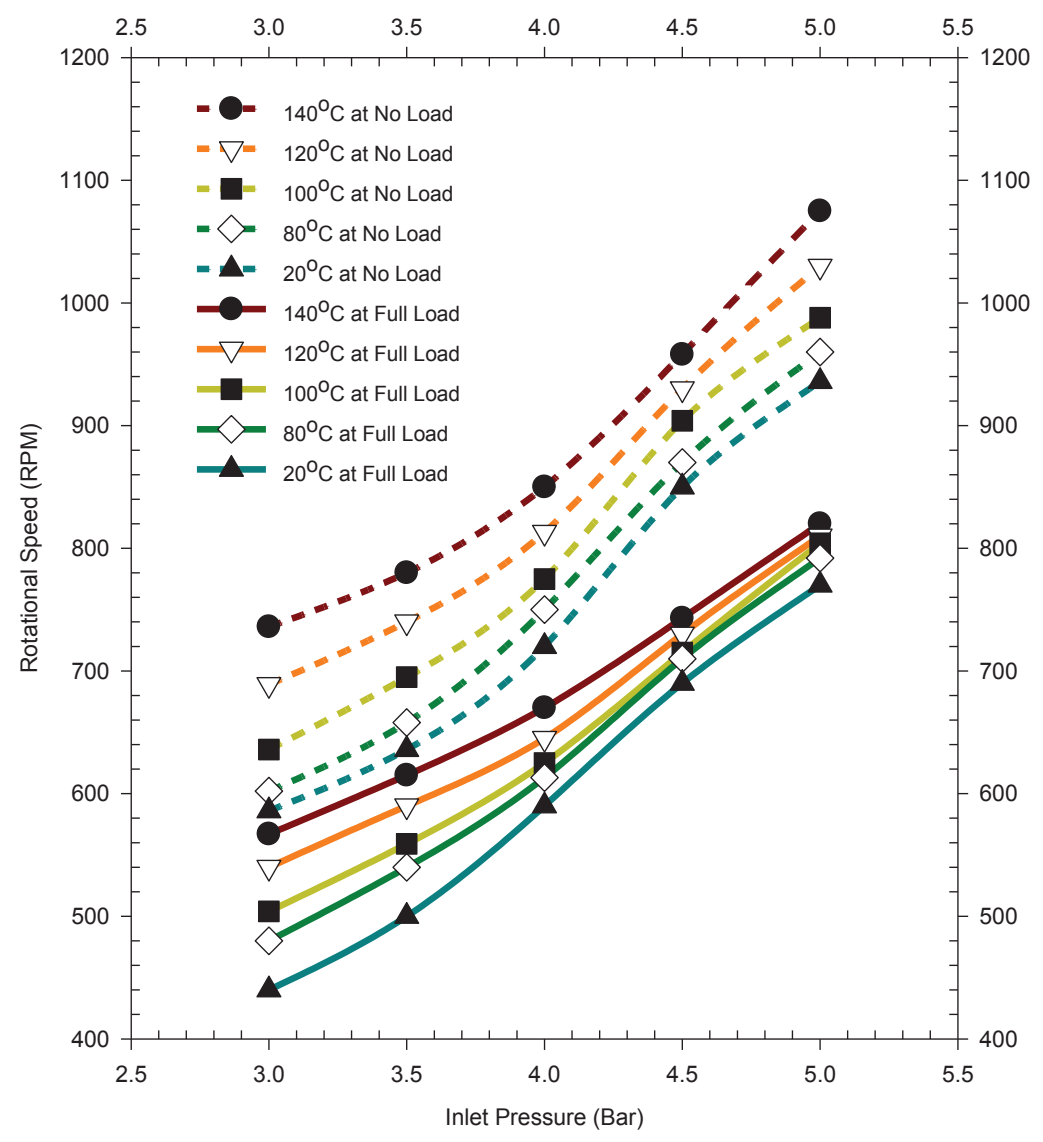

Figure 4. The rotational speed of the RPE at no load and full load conditions with different inlet pressure and temperature conditions.

The same phenomenon has shown by the brake power of the RPE as it was also increased with the increase of the fluid inlet pressure and temperature. The result for the brake power of the RPE shown in figure 5 as it was RPE tested at full load conditions. At the lowest brake power recorded was 6 Watt at 3 bars and $20^{\circ} \mathrm{C}$, and the highest power delivered by the RPE was 27 Watt, recorded at 5 bars and $140^{\circ} \mathrm{C}$. The RPE speed at both conditions was 440 rpm and $820 \mathrm{rpm}$, respectively. It was also observed here that the effect of pressure variations gave significant changes in the RPE brake power conditions compared to the temperature variations due to the direct effect of the pressure to the force applied to produce the brake power. 


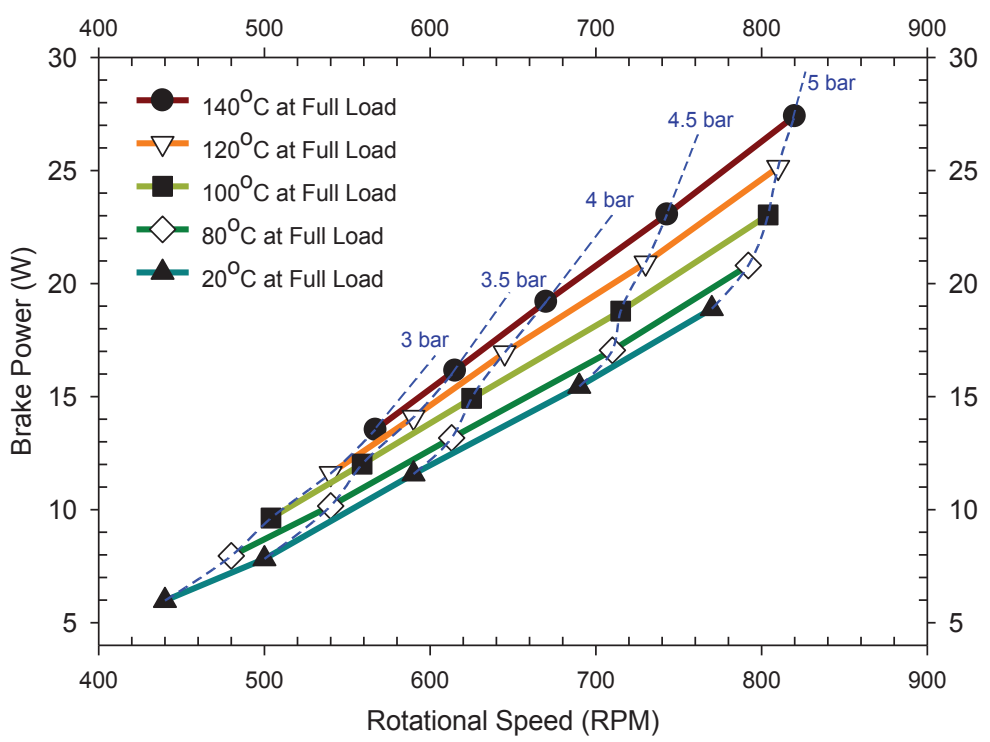

Figure 5. RPE brake power against rotational speed at full load conditions with different inlet temperature and pressure.

Fluid temperature is known to play important roles on the condition of heat capacity and the enthalpy of the fluid, therefore, when the fluid temperature has been increased, the heat capacity of the fluid and its enthalpy also increased, thus, increased the internal work of RPE. In the case of the fluid inlet pressure, the increased in the pressure directly increased the force acted on the RPE piston (Force, $F=$ Pressure, $P \times$ Area, $A$ ) and therefore increased the brake power delivered by the RPE. These reaction mechanisms were closely related to the RPE speed since the internal work and brake power were directly proportional to the rotational speed, and this explains the phenomenon of the increased in fluid inlet temperature and pressure caused the increased in the rotational speed of the RPE as being highlighted above. The study also proved that the developed small-scale dynamometer prototype was able to measure the performance of the RPE although it has been tested up to the maximum conditions, i.e., at full load, and at the highest temperature $\left(140^{\circ} \mathrm{C}\right)$ and pressure (5 bars).

\section{Conclusion}

This study revealed that the performance of RPE was directly proportional to the working fluid inlet pressure and temperature, and proved that the developed small-scale dynamometer prototype was successfully measured the performance of the RPE for the entire testing conditions, even it is still under development. From the study, it was observed that the highest brake power produced by the RPE was 27 Watt when it operated at $140^{\circ} \mathrm{C}$ and 5 bars of inlet fluid, at a rotational speed of $820 \mathrm{rpm}$. The pressure of the inlet fluid can give significant changes on the performance of the RPE due to its direct relation to the RPE actual rotating force.

For the future work, it is strongly suggested that the current valve timing control system should be replaced by the electronic control system that is fast enough to react in order to ease the study especially in terms of valve timing (intake and exhaust). The 
dynamometer prototype that has been developed in this study requires a comprehensive calibration work to ensure to measurement can be done more precisely according to the published standard for the force and torque measurement. Although the dynamometer prototype investigated here was mainly used for RPE performance measurement, this not the only limitation of this dynamometer application. The dynamometer can also be used to measure the performance of any other power generation devices, provided that the power generated is in the form of rotating shaft.

\section{Acknowledgements}

Authors would like to acknowledge the Ministry of Higher Education Malaysia (MOHE) for the financial support given under the Research Acculturation Grant Scheme (RAGS: R056) and Universiti Tun Hussein Onn Malaysia for the facilities and place provided for this research project. Authors also would like to express thank to all individuals and groups for ideas, guidance and assistance contributed during the completion of this research.

\section{References}

[1] Othman M F, Adam A, Naja G, and Mamat R, 2017, "Green fuel as alternative fuel for diesel engine: A review," Renew. Sustain. Energy Rev., vol. 80, no. May, pp. 694-709.

[2] Penkuhn M, and Tsatsaronis G, 2017, "A decomposition method for the evaluation of component interactions in energy conversion systems for application to advanced exergy- based analyses," Energy, vol. 133, pp. $388-403$.

[3] Quoilin S, Declaye S, Tchanche B F, and Lemort V, 2011, "Thermo-economic optimization of waste heat recovery Organic Rankine Cycles," Appl. Therm. Eng., vol. 31, no. 14-15, pp. 2885-2893.

[4] Cavazzini G, Bari S, Pavesi G, and Ardizzon G, 2017, “A multi- fluid PSObased algorithm for the search of the best performance of sub-critical Organic Rankine Cycles," Energy, vol. 129, pp. 42-58.

[5] Zhang X, Wu L, Wang X, and Ju G, 2016, "Comparative study of waste heat steam SRC, ORC and S-ORC power generation systems in medium-low temperature Organic Rankine cycle," Appl. Therm. Eng., vol. 106, pp. $1427-1439$.

[6] Chang J, Hung T, He Y, and Zhang W, 2015, "Experimental study on lowtemperature organic Rankine cycle utilizing scroll type expander," Appl. Energy, vol. 155, pp. 150-159.

[7] Batzer R E, and D D Frey, 2011, "Design and Construction of a Dynamometer," Massachusetts Institute of Technology.

[8] Menon S K, 2006, "Performance Measurement and Scaling in Small Internal Combustion Engines," University of Maryland.

[9] Mohamad M N A, Manshoor B, Hushim M F, Amirnordin S H, and 
Muhammad W N A W, 2016, "Development of twin piston expander with solenoid valve for organic rankine cycle," ARPN J. Eng. Appl. Sci., vol. 11, no. 18, pp. 11154-11158. 